\title{
Interfaz de marketing, operaciones y cadena de suministros: estrategias para servicios
}

\section{Marketing, operations and supply chain interface: services strategies}

\section{INFORMACIÓN DEL}

\section{ARTÍCULO}

Fecha de recepción: 2 de Junio de 2021 Fecha de aceptación: 22 de Octubre de 2021.

1 Máster en Administración de Empresas, Universidad de las Américas. Docente-investigador, Universidad Internacional del Ecuador (UIDE)-Ecuador.

E-mail: manavasre@uide.edu.ec Código ORCID:

https://orcid.org/0000-0001-5808-9050

${ }^{2}$ Máster en Administración de Empresas, Instituto de Estudios Superiores de Instituto de Estudios Superiores de
Administración (IESA). Docenteinvestigador, Universidad Internacional del Ecuador (UIDE)- Ecuador.

E-mail: jacastillode@uide.edu.ec

Código ORCID:

https://orcid.org/0000-0003-0702-7153

CITACIÓN: Navas-Recalde, A., \& Castillo-Cáceres, J. (2021). Interfaz de marketing, operaciones y cadena de suministros: estrategias para servicios. Podium, 40, 1-20.

doi:10.31095/podium.2021.40.1

\section{ENLACE DOI:}

http://dx.doi.org/10.31095/podium.202 1.40 .1

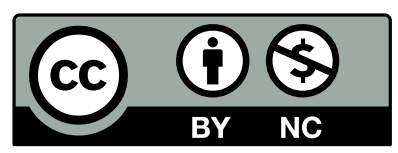

Andrea Navas-Recalde ${ }^{1}$, Janeth Castillo-Cáceres ${ }^{2}$

\section{Resumen}

El propósito de esta investigación es analizar las actividades claves y estrategias en las interacciones de Marketing-Administración de Operaciones y Cadena de Suministro (AOCS), que resultan favorables para la competitividad. Se efectuó una revisión literaria, con diseño cualitativo integral, de artículos científicos desde el año 2009 al año 2021, de acceso abierto, en las bases de datos: WOS, SCOPUS, SCIENCE DIRECT y EMERALD. El alcance se enfocó en actividades claves y estrategias en la interfaz martketing-AOCS (y sus interfaces aisladas), mecanismos para su coordinación y efectos en la competitividad en servicios. Se evidenció que los mecanismos de alineación de las interfaces, por lo general, dependen de la organización y sus interacciones con el mercado; que no existen estudios de la interfaz marketing-AOCS; y que las posibles sinergias de marketing con otras funciones pueden generar mayor competitividad en la empresa debido a la alineación eficiente de esta interfaz.

\section{Palabras Clave:}

Interfaz, marketing, operaciones, cadena de suministro, AOCS, alineación interfuncional, servitización.

Clasificación JEL: M310, L223.

\begin{abstract}
The purpose of this research is to analyze the key activities and strategies in Marketing-Operations Management and Supply Chain AOCS (Spanish acronym) interactions, which are favorable for competitiveness. A literary review was carried out, with a comprehensive qualitative design, of scientific articles from 2009 to 2021, using open access, and other databases such as WOS, SCOPUS, SCIENCE DIRECT and EMERALD. The scope of the investigation was focused on: key activities and strategies in the marketing-AOCS interface (and its isolated interfaces), mechanisms for their coordination and effects on competitiveness, in services. It was concluded that the interface alignment mechanisms usually depend on the organization and its interactions with the market. In addition, the study concluded that there are no studies on marketing-AOCS interface; and that the possible synergies between marketing and other functions can generate greater competitiveness in the company due to the efficient alignment of this interface.
\end{abstract}

Keywords:

Interface, marketing, operations, supply chain, OMSC, cross-functional alignment servitization.

JEL Classification: M310, L223.

$$
1
$$

PODIUM No. 40, Diciembre 2021, pp. 1-20

(C) Universidad Espíritu Santo - UEES

ISSN: 1390-5473 e-ISSN: 2588-0969 


\section{Introducción}

Aunque el marketing y las operaciones están claramente conectados, estas áreas operativas y estratégicas de las empresas, recientemente se han integrado de forma más estrecha, $\mathrm{y}$ ahora se conoce que esta integración es muy importante para la ventaja competitiva de una empresa; pues se ha demostrado que influye positivamente en los indicadores de rendimiento, como la productividad de la planta, el rendimiento de la inversión (ROI) y el desempeño financiero (Sombultawe, 2017).

En las organizaciones de servicio, la coordinación de marketing-operaciones juega un rol de mayor preponderancia que en las empresas de producción tradicional, ya que el mismo cliente se constituye en proveedor (sus opiniones, información, acciones). Si bien, en un proceso de producción los proveedores reciben pago por sus insumos; en los servicios los clientes, que son a su vez proveedores, son quienes generan el pago. Por ello, marketing y operaciones deben trabajar estrechamente para procesar y entregar al cliente un servicio que pueda valorar (Dixon, Karniouchina, van der Rhee, Verma, y Victorino, 2014) y que a su vez represente un insumo valioso para la organización.

La evolución en la estrategia de servicios puede ser comparada con procesos de cambios y mejoras en ofertas, capacidades y procesos para que la estrategia de la empresa se centre en ofertar un servicio en el que el producto esté implícito. De hecho, los productos con un alto contenido de servicios se conocen como servitización, lo que describe la adición de servicios a las ofertas de productos principales de los fabricantes para crear valor al cliente (Raddats, Kowalkowski, Benedettini, Burton y Gebauer, 2019).

Sombultawee y Boon-itt (2019) realizaron una revisión de la literatura y encontraron que hay tres dominios principales en la investigación de interfaces de operaciones y marketing, estos son: la alineación entre estas dos áreas, la identificación de variables que impactan a la empresa y el análisis del efecto de la interfaz. Zanon, Filho, Jabbour, y Jabbour (2013), indican que el término "interfaz" lo utilizan los investigadores en referencia a interacciones entre funciones $y$ esa misma connotación se usa en el presente estudio.

Golgeci y Gligor (2017), en su investigación, indican que la noción general de posibles sinergias entre marketing y otras funciones ha tenido un subyacente hilo conductor, en el cual marketing puede desarrollar y aprovechar sinergias con diversas funciones dependiendo de: la estructura y el tipo de la empresa, dinámicas intraorganizacionales y otras fuerzas externas. Sin embargo, ha faltado una mayor evaluación de la interacción entre el marketing específico y las capacidades de Gestión de la Cadena de Suministro (GCS); importantes para las empresas dentro de su contexto organizacional.

Considerando que esta noción de 
posibles sinergias entre marketing y otras funciones puede ser más eficaz en la alineación de la interfaz marketingAOCS (Administración de Operaciones y Cadena de Suministro), en lugar de interfaces aisladas marketing-operaciones y marketing-GCS; en el presente artículo se efectuó una revisión literaria donde se analizaron y consolidaron los resultados de investigaciones anteriores de la interfaz marketing-AOCS (incluyendo sus interfaces aisladas y consideraciones durante el distanciamiento social por Covid 19), con el objetivo de analizar las actividades claves y estrategias presentes en dicha interfaz, mecanismos para propiciar su coordinación y efectos en la competitividad; para que puedan ser considerados como mejores prácticas en la competitividad empresarial.

\section{Revisión de literatura}

Actividades claves y estrategias en la interfaz marketing-AOCS

Según Chase y Jacobs (2019, p.4):

Por operaciones se entienden los procesos de manufactura y procesos de servicio mediante los cuales los recursos de una empresa se transforman en los productos deseados por los clientes...Una cadena de suministro se refiere a procesos que desplazan información y material con destino y origen en los procesos de manufactura y servicio de la empresa.

Con base en la anterior definición, en el presente artículo se analizó la interfaz marketing-AOCS, y sus interfaces aisladas, en especial en servicios, con la finalidad de estudiar las actividades claves y estrategias presentes en dicha interfaz que resultan favorables para la competitividad.

En la Tabla 1 se consolida la información de la revisión literaria sobre las estrategias y temas claves en la interfaz de marketing-AOCS, y sus interfaces aisladas, clasificados por categorías.

\section{Marketing-operaciones}

Bitner (1992), quien introdujo el término Servicescapes, refiriéndose al entorno físico de la prestación de servicios y su impacto en el comportamiento de clientes y empleados, explora también la habilidad del manejo del entorno físico para facilitar el logro de objetivos organizacionales y de mercadeo. Generalmente, el servicio se produce y consume al mismo tiempo, por lo que el cliente está experimentando el servicio dentro del mismo espacio físico donde se presta, lo que puede tener un fuerte impacto en sus percepciones sobre dicha experiencia y en su satisfacción final.

Zomerdijk, y Voss (2009), indican que los elementos tangibles del servicio, o donde se crea la experiencia, se considera influyen en las percepciones y el comportamiento del cliente. Así, el entorno físico puede diseñarse para evocar emociones y respuestas particulares, y su gestión eficaz es vital para la creación de un servicio convincente. 
Tabla 1 .

Estrategias y temas claves en la interfaz de marketing-AOCS

\begin{tabular}{|c|c|c|}
\hline Autores & Estrategias y temas claves & Observaciones \\
\hline \multicolumn{3}{|l|}{ Marketing-operaciones } \\
\hline Bitner (1992) & $\begin{array}{l}\text { Servicescapes - Diseño de entorno de } \\
\text { servicio }\end{array}$ & $\begin{array}{l}\text { Configuración física del ambiente donde se } \\
\text { presta un servicio de la empresa, lo que } \\
\text { implica la interacción marketing - } \\
\text { operaciones. }\end{array}$ \\
\hline Zanon et al. (2013) & Marketing-operaciones & $\begin{array}{l}\text { Las actividades asociadas con la } \\
\text { investigación conjunta sobre el contexto } \\
\text { competitivo, la comprensión de los costos - } \\
\text { beneficios interfuncionales que permiten } \\
\text { reducir el conflicto y la comprensión de las } \\
\text { necesidades del cliente y el desempeño } \\
\text { operativo; fomentan la alineación en la } \\
\text { interfaz. }\end{array}$ \\
\hline Dixon et al. (2014) & $\begin{array}{l}\text { Decisiones gerenci ales de operaciones y } \\
\text { de marketing interrelacionadas }\end{array}$ & $\begin{array}{l}\text { Referente a: diseño del servicio, desarrollo } \\
\text { de nuevos productos, inventario, Cadena de } \\
\text { Suministro (CS) y administración logística, } \\
\text { pensamiento lean, competencia basada en } \\
\text { el tiempo, gestión de línea de espera, } \\
\text { estandarización y control de procesos, } \\
\text { personalización masiva y co -creación, } \\
\text { sostenibilidad y responsabilidad social } \\
\text { corporativa, entre otras. }\end{array}$ \\
\hline $\begin{array}{l}\text { Shahid y Khan } \\
\text { (2016) }\end{array}$ & $\begin{array}{l}\text { Pedidos telefónicos y servicios de } \\
\text { entrega de comida rápida }\end{array}$ & $\begin{array}{l}\text { Las operaciones y estrategias de marketing } \\
\text { deben combinarse cuidadosamente para } \\
\text { que la empresa cumpla o supere las } \\
\text { expectativas propuestas por marketing. }\end{array}$ \\
\hline $\begin{array}{l}\text { Samuel, Mesak e } \\
\text { Inman (2017) }\end{array}$ & Introducción de nuevos productos & $\begin{array}{l}\text { Integración de las funciones de marketing y } \\
\text { operaciones. }\end{array}$ \\
\hline $\begin{array}{l}\text { El Ouardighi, } \\
\text { Feichtinger y } \\
\text { Fruchter }(2018)\end{array}$ & $\begin{array}{l}\text { Acelerar la difusión de innovaciones de } \\
\text { boca en boca mixto (es decir, positivo y } \\
\text { negativo) a través de la interacción } \\
\text { marketing-operaciones }\end{array}$ & $\begin{array}{l}\text { La participación del marketing está } \\
\text { relacionada con el precio y el esfuerzo } \\
\text { publicitario. En la gestión de operaciones se } \\
\text { refiere a la calidad del diseño y la } \\
\text { conformidad de la calidad. }\end{array}$ \\
\hline $\begin{array}{l}\text { Sombultawee y } \\
\text { Boon-itt (2018) }\end{array}$ & $\begin{array}{l}\text { Actividades y procesos que podrían estar } \\
\text { presentes dentro de los puntos de } \\
\text { alineación (marketing -operaciones) }\end{array}$ & $\begin{array}{l}\text { Integración de la planificación estratégica, } \\
\text { pronósticos estratégicos, desarrollo de } \\
\text { nuevos productos, procesos y servicios; } \\
\text { pronósticos tácticos, planificación de } \\
\text { operaciones y ventas (gestión de la } \\
\text { demanda) e integración operativa. }\end{array}$ \\
\hline $\begin{array}{l}\text { Vicenzi, Antoni, } \\
\text { Damacena, Riss y } \\
\text { Manosso (2020) }\end{array}$ & $\begin{array}{l}\text { Marketing y operaciones en innovación } \\
\text { de producto }\end{array}$ & $\begin{array}{l}\text { La integración de estas áreas tiene un } \\
\text { impacto significativo en el éxito de la } \\
\text { innovación. El intercambio de información } \\
\text { en las etapas inicial y final del proceso de } \\
\text { innovación de productos es un motor para } \\
\text { lograr un mejor desempeño. }\end{array}$ \\
\hline Bijmolt, et al. (2021) & Modelo de Venta Omnicanal & $\begin{array}{l}\text { Considera en cada decisión de diseño } \\
\text { omnicanal un enfoque dual del lado de la } \\
\text { demanda (marketing) y del lado de la oferta } \\
\text { (operaciones). }\end{array}$ \\
\hline
\end{tabular}

4

PODIUM No. 40, Diciembre 2021, pp. 1-20

(C) Universidad Espíritu Santo - UEES

ISSN: 1390-5473 e-ISSN: 2588-0969 


\begin{tabular}{lll}
\hline Autores & Estrategias y temas claves & Observaciones \\
\hline Finger et al. (2021) & Marketing-operaciones & Mediante el análisis de datos de la realidad \\
& & operativa de una empresa brasileña de baja \\
& intensidad tecnológica, se obtuvo que las \\
& decisiones sobre las dimensiones del \\
& marketing de lugar, canal, precio y \\
& producto; ejercen un impacto significativo \\
& en el desempeño de las dimensiones de \\
& flexibilidad entrega del área de operación. \\
\hline
\end{tabular}

Marketing-GCS

Pero y Lamberti Marketing-GCS (2013)

Altuntas (2015) Gestión Sustentable de la Cadena de Demanda (GSCD)
El desarrollo de nuevos productos representa un momento de la verdad en la interfaz marketing-GCS.

Involucra la información de mercado con las actividades de la Gestión de la Cadena de Suministro Sostenible (GCSS) y alinea estas actividades con las expectativas del cliente con respecto a la sostenibilidad. La cadena de demanda es una perspectiva que defiende mejorar la interacción entre las funciones de marketing y la GCS .

\begin{tabular}{ll}
$\begin{array}{l}\text { De Souza, Giro y } \\
\text { Caldeira (2016) }\end{array}$ & GCS verde \\
\hline $\begin{array}{l}\text { Bhoompag, Sambrani } \\
\text { y Prabhukumar } \\
(2017)\end{array}$ & Marketing verde \\
\hline $\begin{array}{l}\text { Golgeci y Gligor } \\
(2017)\end{array}$ & Marketing-GCS \\
\hline
\end{tabular}

Indica que el marketing verde media, en parte, la relación entre la GCS y el rendimiento empresarial.

Modificaciones en la gama de productos, alteración del proceso de producción y otras actividades.

Identifican la capacidad de innovación y aprendizaje de mercado como capacidades claves de marketing, y la agilidad de la CS y la capacidad relacional, como capacidades claves de GCS. Las relaciones entre estas capacidades de marketing estratégico y SCM siguen un patrón.

\begin{tabular}{|c|c|c|}
\hline Noci (2019) & Marketing-GCS & $\begin{array}{l}\text { Las estrategias de marketing deben ser } \\
\text { capaces de reconfigurar las cadenas de } \\
\text { suministro para remodelar el valor para los } \\
\text { clientes. }\end{array}$ \\
\hline Veeralakshmi (2020) & Marketing ecológico & $\begin{array}{l}\text { Protección del bienestar a largo plazo de los } \\
\text { consumidores y la sociedad mediante la } \\
\text { producción y el uso de productos puros. }\end{array}$ \\
\hline $\begin{array}{l}\text { Gölgeci y } \\
\text { Kuivalainen (2020) }\end{array}$ & Alineación marketing-GCS & $\begin{array}{l}\text { Favorece a la Cadena de Suministro } \\
\text { Resiliente (CSR) y el desempeño. }\end{array}$ \\
\hline Attia (2015) & $\begin{array}{l}\text { CS triple A (ágil, adaptable y alineada) } \\
\text { y; alineación con la estrategia de } \\
\text { marketing }\end{array}$ & $\begin{array}{l}\text { En empresas que trabajan en la industria } \\
\text { textil egipcia, el desempeño organizacional } \\
\text { está asociado positivamente con el } \\
\text { desempeño de la CS, y este se ve afectado } \\
\text { directamente por la CS triple A y su } \\
\text { alineación con la estrategia de marketing. }\end{array}$ \\
\hline
\end{tabular}

5

PODIUM No. 40, Diciembre 2021, pp. 1-20

(C) Universidad Espíritu Santo - UEES

ISSN: 1390-5473 e-ISSN: 2588-0969 
Andrea Navas-Recalde, Janeth Castillo-Cáceres

Marketing-AOCS durante el Distanciamiento Social

\begin{tabular}{lll}
\hline Autores & Estrategias y temas claves & Observaciones \\
\hline Wang., Hong, Li, y & $\begin{array}{l}\text { Nuevos valores: medidas de bioseguridad } \\
\text { Gao (2020)/ He y }\end{array}$ & $\begin{array}{l}\text { La pandemia (Covid 19), obligó a } \\
\text { implementar innovaciones en sistemas, } \\
\text { Harris (2020) }\end{array}$
\end{tabular}

Fuente: Elaboración propia.

En el diseño del servicio se procura que los encuentros con el cliente sean lo más favorables posibles para el negocio, $\mathrm{y}$ en ello estas dos disciplinas (marketing-operaciones) confluyen una vez más (Dixon et al., 2014).

Dentro de las interacciones marketing-operaciones en servicios, se hallan las que Dixon et al. (2014) encontraron en algunas publicaciones, las cuales tienden a aceptar que las prioridades operacionales competitivas deberían posicionarse y alinearse con las prioridades de comercialización (precio, producto, lugar y promoción); es decir, deben apuntar a lo mismo.

En cuanto al desarrollo de nuevos productos, una interacción marketingoperaciones es que las decisiones del diseño de productos y servicios deben basarse en las capacidades y restricciones de comercialización y producción; esto con la finalidad de potenciar la coordinación (Dixon et al., 2014). Adicional, se encuentra el intercambio de información en las etapas inicial y final del proceso de innovación de productos como motor para lograr un mejor desempeño (Vicenzi et al., 2020).

Por lo tanto, si se habla de diseño de experiencias (Dixon et al., 2014), la relación entre marketing-operaciones es aún más importante considerando que se diseña y ejecuta una oferta de servicios centrada en la experiencia del cliente.

\section{Marketing-GCS}

Pero y Lamberti (2013), manifiestan que la interfaz entre marketing-GCS ha interesado por mucho tiempo a la academia y los profesionales, y que la causa de este interés es doble: por un lado, la propia definición de GCS como integración del suministro (logística y operaciones) y gestión de la demanda (marketing) dentro y entre empresas, abarca muchos fenómenos y prácticas comunes a la disciplina, práctica y gestión de marketing; por otro lado, a menudo la GCS tienen un trasfondo de operaciones y la interfaz con marketing se ve afectada por los mismos problemas que la interfaz de marketing-operaciones; generando frecuentes conflictos que a menudo resultan en un disminución de la eficacia, eficiencia y flexibilidad.

La CS debe estar integrada y alineada con las operaciones externas de clientes, proveedores y otros miembros del canal, construyendo relaciones estratégicas entre ellos. Las capacidades del área de marketing son útiles para obtener información sobre los distintos socios de la CS (Yu, Jacobs, Chávez, y Feng, 2017). En el marketing de servicios, por ejemplo, existe la necesidad imperiosa de comprender mejor al cliente y desarrollar, 
con base en ese entendimiento, relaciones basadas en confianza e integración de recursos (Vorre, 2019).

Una estrategia muy usada en la actualidad, para servicios, es la omnicanalidad la cual requiere que las empresas coordinen sus actividades en las diferentes etapas del recorrido del cliente, del flujo de productos, y en todos los canales; lo que conlleva que estas adopten un enfoque integrador; considerando en cada decisión de diseño omnicanal un enfoque dual del lado de la demanda (marketing) y del lado de la oferta (operaciones), con áreas clave de decisión como: surtido e inventario, distribución, entrega y devoluciones (Bijmolt, et al., 2021).

La estrategia omnicanal difiere del multicanal porque se centra en que los clientes reciban una oferta integrada y experiencia única pensada en la marca más que en el canal. Esta estrategia se centra en el diseño, implementación, coordinación y evaluación de los diferentes canales; siendo su enfoque la gestión y optimización del rendimiento de cada canal (Ailawadi y Farris, 2017).

O'Connor, Lowry, y Treiblmaier (2020), se centraron en optimizar el rendimiento de la CS mediante la contratación de personal, específicamente para el intercambio de información, coordinación de múltiples proveedores mediante seguimiento de rutinas que fomenten la cooperación y que como resultado optimicen varios factores de costos, gestión de inventario $\mathrm{y}$ distribución.
La integración de la CS es un proceso progresivo, que debe ser trabajado en diferentes etapas de relación, donde las empresas servitizadas tienen un mayor nivel de innovación (Freije, de la Calle, y Ugarte, 2021). Según O'Connor et al. (2020), la estrategia de aumentar la colaboración con proveedores refuerza la capacidad de respuesta y por ende la innovación y rentabilidad, lo que ayuda a la sostenibilidad a largo plazo de las CS globales, estas relaciones entre actores humanos, como la interacción entre proveedores y compradores radican en bases conceptuales como la del marketing de servicios, enfocado en el mejoramiento de dichos vínculos.

Altuntas (2015) propone la estrategia de la GSCD, como un esfuerzo para involucrar la información de mercado con las actividades de la GCSS y alinear estas actividades con las expectativas del cliente con respecto a la sostenibilidad.

Al implementar la GCS verde, por otra parte, las empresas esperan no solo una mejora en el desempeño ambiental, sino también reconocimiento de imagen corporativa, ventaja competitiva y exposición al mercado; reconociendo la importancia de la conexión GCSmarketing (De Souza et al., 2016, p.189). Las empresas buscan generar reputación basadas en su CS, siendo que esta es considerada como un activo intangible de la percepción del comprador sobre el proveedor, la que sí es correctamente trabajada puede traducirse en una ventaja competitiva de la empresa (Leppelt, Foerstl, y Hartmann, 2013). 


\section{Marketing-AOCS durante el Distanciamiento Social}

La pandemia de COVID- 19, por su severidad, generó una interrupción nunca vista en cuanto a la operación de la CS, afectando a todos sus actores tanto mayoristas como minoristas, haciendo evidente la importancia en la resiliencia de la gestión de suministro (Chowdhury, Sarkar, Saha, y Anik, 2020).

Dicha situación, desafió a las empresas a tomar acciones inmediatas con el objetivo de sobrevivir en un mercado casi desconocido por sus nuevas necesidades y preferencias, actuar sobre lo incierto y tomar decisiones apresuradas y previsoras; obligando a desarrollar agilidad estratégica para implementar innovaciones en sistemas, operaciones, $\mathrm{y}$ tácticas que les permita llegar a los clientes (He y Harris, 2020); y así mantenerse de pie en una guerra donde muchos fueron derrotados por su incapacidad de renovarse.

Debido al COVID-19, las empresas manufactureras han experimentado interrupciones graves en la CS lo cual ha sido llamado "cisne negro" donde la resiliencia de las organizaciones juega un papel primordial para una eficiente GCS (Yang, Xie, Yu y Liu, 2020) y un adecuado servicio al cliente. En el estudio realizado por Lozano y Barreiro-Gen (2021), obtuvieron que las empresas, para ser más resilientes, deben contar con sistemas organizacionales. Estos, de preferencia, deben ser interdepartamentales, donde los equipos de ventas y marketing pueden alinear sus objetivos y crear un marco común, que facilite el intercambio de ideas (Le y Massey, 2019).

Si bien, antes un valor importante en la generación de servicio era el ambiente que un determinado proveedor de servicio pudiera ofrecer (Kwok, Tang \& Yu, 2020); hoy lo más valorado por los consumidores es: que un lugar cuente con las medidas de bioseguridad, y la posibilidad de una oferta en línea. Las empresas deben prestar más atención en cómo lograrlo apoyados de la generación de innovación para ofrecer un valor superior al cliente (Wang et al., 2020).

Es así como, desde el punto de vista centrado en el usuario, se integran las necesidades y las experiencias de estos para la generación de sistemas de información interactivos que ayuden a comprender y dinamizar los distintos roles, tanto de los empleados como de los clientes, para el desarrollo de innovación en la experiencia del usuario (Prestes, Teixeira, Patricio, y Sangiorgi, 2019).

En este contexto, el e-marketing se ha convertido en una gran oportunidad para que los especialistas en marketing respondan y gestionen esta crisis (Patel y Malpani, 2020, p. 1778). Muchas empresas que no hicieron la transición digital han perdido todo el acceso a los clientes y es menos probable que sobrevivan a la pandemia (Kim, 2020, p. 215). La evolución de la globalización y el aumento de las actividades de comercio exterior han dado al sector logístico una importancia estratégica (Akdoğan y Durak, 2016, p. 576) 
apoyándose desde distintas aristas en el e-marketing.

Los microambientes de marketing han sido afectados por una serie de cambios, muchas de estas alteraciones probablemente generen una nueva forma de trabajo y de comportamiento del consumidor (He \& Harris, 2020; Kwok, et al., 2020; y Wang et al., 2020). Lo que podría producir nuevas oportunidades de encuentro para la interfaz marketingAOCS.

\section{Mecanismos para propiciar la coordinación de Marketing-AOCS}

El grado de coordinación entre las actividades de marketing y operaciones a nivel estratégico, táctico y operativo varía significativamente entre empresas. No hay evidencia suficiente sobre los beneficios de su alineación para satisfacer al cliente (Sombultawe, 2017). Las estrategias de marketing son realmente efectivas solo cuando la empresa cuenta con los recursos para cumplir con las promesas que hacen a los clientes a través de sus campañas (Sombultawee y Boon-itt, 2018, pp. 1-2).

El marketing y las operaciones como áreas funcionales representan áreas clave de valor agregado de la empresa comercial moderna (Samuel et al., 2017). Según Sombultawee y Boon-itt (2018, p.8), la alineación marketing-operaciones no se produce orgánicamente, especialmente en una estructura organizacional jerárquica departamental. Ellos indican que, son los gerentes quienes deben deliberadamente crear canales de comunicación y decisión, formales e informales; que faciliten la coordinación de actividades y toma de decisiones en los niveles estratégico, operacional y táctico. Lo que se podría lograr empoderando equipos ambidiestros desde el ámbito comercial y de operaciones para mantener el equilibrio utilizando procesos autónomos (Goh y Eldridge, 2019).

Según O'Connor et al. (2020), para una comunicación proactiva, se requiere: un cierto nivel de confianza y compromiso relacional, que ayuden a mejorar procesos claves, desarrollar nuevos productos y mercados. Goh y Eldridge (2019) rescatan algunos mecanismos de coordinación marketingoperaciones que pueden explicar las formas en que las organizaciones coordinan su trabajo, como son: mutuo ajuste, supervisión directa, estandarización de procesos de trabajo, estandarización de los resultados del trabajo y estandarización de las habilidades de los trabajadores.

Según el estudio de Samuel et al. (2017), en el análisis de sensibilidad hecho entre los campos relacionados de marketing y operaciones, se revelan las mayores ganancias y un ritmo más rápido de introducción de nuevos productos, lo que generalmente se asocia con un parámetro de elasticidad de precio más bajo, una velocidad de difusión más rápida, un mayor potencial de mercado, productos de consumo más costosos y un menor costo de introducción de nuevos productos.

Entre otras interacciones interesantes 
a ser trabajadas entre marketing $\mathrm{y}$ operaciones está el hecho de que las operaciones pueden centrarse en la gestión de costes, decisiones con impacto en el tiempo de entrega (Marques, Lacerda, Riehs, y Teixeira, 2014). Así también; según Fu, Cheng y Yi (2019); el precio de transferencia juega un papel importante en la dirección de la toma de decisiones, así como en coordinar los beneficios del departamento de operaciones y el de marketing de la empresa.

Ávila, Lima, Moreira, Pires y Bastos (2019), consideran que la planificación de ventas y operaciones (PVO) produce ahorros significativos a nivel operacional y mayor efectividad en el plan de demanda de la empresa, mejora la comunicación y el flujo de información entre los diferentes departamentos que fomentan la alineación (marketing, producción y $\mathrm{CS}$ ) y resolución rápida de problemas con mayor esfuerzo de trabajo en equipo.

Según los resultados obtenidos por Le y Massey (2019), estructurar las ventas y el marketing como una sola unidad y crear funciones multifuncionales de los equipos de proyecto, mejoran la interfaz. Así también, la rotación de puestos y el establecimiento de funciones cruzadas reduce el conflicto y aumenta la colaboración entre ventas y marketing lo cual influye positivamente en el rendimiento empresarial.

Este pensamiento lo comparte Piercy (2010), quien menciona que el proceso de reunir a las personas de manera informal y formal también es importante para alentar mejor la comprensión del personal; así también, su rotación y el uso de equipos interfuncionales en la planificación y resolución de problemas pueden ser herramientas poderosas para compartir conocimientos.

La alineación de los sistemas de incentivos y recompensas se identificó como una de las principales herramientas utilizadas para promover la cooperación entre departamentos, junto con las medidas basadas en indicadores, aunque haya indicios de que estas pueden ser inadecuadamente implementadas, en el sentido de que se usan con relativa poca frecuencia y son medidas de cooperación indirectas basadas en resultados (Sombultawee y Boon-itt, 2020).

Piercy (2010) considera la necesidad de: tener una estrategia sólida para impulsar el avance del negocio e involucrar a todas las áreas en la formación de dicha estrategia y proporcionar así un enfoque unificador por encima de metas y objetivos funcionales individuales; además, expone que los sistemas de recompensa y evaluación deben respaldar la integración.

Bardhan y Pattnaik (2017), indican que: la inversión en un sistema de información que fomente la comunicación resultaría en mejores productos, la integración en la toma de decisiones también es muy importante, $\mathrm{y}$ la planificación es una función donde el alcance de la integración es mayor. Sostienen que: debe fomentarse la integración, la estrategia y la 
planificación conjunta; además, si las decisiones gerenciales se toman en un entorno integrado, los gerentes estarían expuestos a perspectivas más allá de sus silos funcionales lo que debería conducir a mejores pronósticos, procesos de mejor desempeño y clientes más satisfechos.

Bijmolt et al. (2021), describen oportunidades para aliviar las tensiones que se pueden presentar (en la interfaz marketing-operaciones) haciendo énfasis en el uso de tecnologías de la información, inteligencia artificial y análisis de datos que permita tener información integrada para la toma de decisiones.

Kwortnik y Thompson (2009, p. 18), visualizaron la Gestión de la Experiencia del Servicio como una nueva área funcional lo suficientemente alta en la jerarquía organizacional para que marketing y operaciones le reporten, en este sentido sugieren la elevación de esta área en la organización porque abogaría por el cliente y el equipo de operaciones para garantizar que estos clientes obtengan lo que quieren, dadas las capacidades operativas realistas.

Zanon et al. (2013), en cuanto a la interfaz marketing-operaciones hallaron que las actividades asociadas con la investigación conjunta sobre el contexto competitivo parecen motivar al personal directivo a comprender las amenazas y oportunidades en los negocios, así como, que la comprensión de los costos-beneficios interfuncionales permiten reducir el conflicto mediante el intercambio de opiniones y valores que condicionan una distribución de recursos más adecuada y participativa, y además que la comprensión de las necesidades del cliente y el desempeño operativo también apoyan en fomentar la alineación en esta interfaz.

Según Noci (2019), la evolución de los mercados hacia una lógica de servicio dominante exige ser impulsadores del mercado, donde las estrategias de marketing deben ser capaces de reconfigurar las CS para remodelar el valor de los clientes. Esto implica un cambio organizativo, en el cual la evolución de la interfaz entre marketing-GCS recae primero en la organización, incluso antes del proceso o la tecnología, con un peso creciente en el diseño y el despliegue de una arquitectura efectiva de interacciones con el mercado.

Alineación entre Marketing-AOCS, su efecto en la competitividad

El conocer el ambiente de fabricación, así como los entornos externos, es un facilitador clave para las estrategias centradas en el consumidor; donde las iniciativas de gestión de calidad fomentadas por grupos de trabajo multifuncionales entre operaciones y marketing, al lograr acuerdos en el equipo, generan un mejor acercamiento con las necesidades del cliente y aumento del rendimiento organizacional (Maynard, Falcone, Petersen, Fugate y Bonney, 2020).

Gölgeci y Kuivalainen (2020) obtuvieron, en su estudio, que tanto la capacidad de absorción como la 
alineación de la GCS y el marketing son necesarias para realizar el valor real del capital social para la CSR y el desempeño. Yu, Ramanathan y Nath (2014) obtuvieron, para el sector minorista del Reino Unido, que la capacidad de marketing tiene un efecto positivo significativo en la de operaciones, y la capacidad operativa está relacionada significativa y positivamente al desempeño financiero.

Es interesante notar que, la alineación marketing-operaciones, siendo un tema que ha llamado tanto la atención de personal de la industria, como de investigadores y académicos; en la práctica su aplicación ha quedado corta y aunque se han discutido varios términos, como el ajuste, la interfaz y la cooperación, nunca ha existido una teoría subyacente sólida sobre cómo se produce esta alineación y qué influye en sus prioridades y prácticas (Sombultawee y Boon-itt, 2018, p.8).

En general, el papel del marketing de servicios y de operaciones debe ser altamente enfatizado dentro de la organización y abordado por el más alto nivel de liderazgo. Lo que no se comprende aún es si este nivel de colaboración genera adicionales distracciones y presión sobre los gerentes; por lo que se requiere investigar todavía sobre este tipo de toma de decisiones (Dixon et al., 2014).

Attia (2021) prueba el efecto, en el desempeño de la CS y desempeño organizacional, de alinear la estrategia de mercado en toda la CS. Por lo que la alineación de estas áreas tiene un efecto final sobre el desempeño de la organización. También describe que lograr la alineación de la estrategia de marketing requiere colaboración $\mathrm{y}$ coordinación entre los socios de la CS y el personal de marketing de la organización, y que la posición competitiva de la empresa se verá afectada por el desempeño de esta CS. Sus hallazgos indican que el efecto directo del desempeño de la CS está en el desempeño operativo y estratégico y no directamente relacionado con rendimiento financiero.

Golgeci y Gligor (2017) encontraron que: la alineación de objetivos y actividades organizacionales, la conciencia interfuncional, el adoptar un enfoque holístico para la creación y entrega de valor, el equilibrio de poder entre marketing-GCS y la racionalización de las actividades y de las estructuras organizativas; como representantes de los mecanismos integradores; juegan un papel sutil y fundamental en la facilitación de la sinergia entre la innovación, capacidad de aprendizaje de mercado, agilidad de la CS y capacidad relacional. Dada la naturaleza de la propuesta de valor central de una empresa, estas no pueden separar el desarrollo e implementación de sus capacidades de marketing del desarrollo de sus capacidades en la GCS, para así proporcionar una fuente de ventaja competitiva.

\section{Metodología}

En el presente artículo se efectuó una 
revisión literaria con diseño cualitativo integral: "El proceso consiste en descubrir categorías (patrones) que se encuentran en un conjunto de datos cualitativos y vincularlas para ... desarrollar una teoría; o bien, resolver algún problema..." (Hernández-Sampieri, Cuevas, Mendoza, y Méndez, 2017; pp. 117-118), donde se analizaron e integraron los resultados de investigaciones sobre la interfaz marketing-AOCS, y sus interfaces aisladas; con el objetivo de analizar las actividades claves y estrategias presentes en dicha interfaz y que resultan favorables para la competitividad.

El alcance de la investigación se centra en actividades claves y estrategias en la interfaz martketing-AOCS, y sus interfaces aisladas, mecanismos para propiciar la coordinación en la interfaz, y efectos en la competitividad.

Se eligieron artículos de acceso abierto de las bases de datos: WOS, SCOPUS, SCIENCE DIRECT y EMERALD; desde el año 2009 hasta el año 2021. Fueron seleccionados de manera cronológica descendente aquellos artículos que poseían en su título información relevante para el estudio, de acuerdo con el alcance definido, para posteriormente descartar aquellos que no correspondían. Los términos de búsqueda consideraron: marketing-operations, marketing-supply chain, marketingoperations-supply chain, solos y con el operador booleano and, y las palabras interface ó alignment.

Adicional, se seleccionó un artículo seminal, publicado por el mayor exponente del concepto servicescapes (Bitner, 1992), un libro de operaciones y cadena de suministro de autores reconocidos en dicha materia (Chase y Jacobs, 2019), y una disertación doctoral (Sombultawe, 2017); por su relevancia para el estudio. No se incluyeron artículos relacionados con la industria médica (hospitales).

\section{Resultados y discusión}

Para servicios, el área de operaciones debe prestar atención al diseño de experiencias, el entorno físico y la CS; lo que repercute, en el grado de satisfacción del cliente; siendo de vital importancia cuidar la relación marketing-AOCS para cumplir las promesas realizadas, considerando la capacidad de atención con que se cuente, y que marketing se ajuste a ello en sus promesas, tal como lo exponen: Dixon et al. (2014), Freije et al. (2021), Sombultawe (2017), Sombultawee y Boon-itt (2018), y Zomerdijk, y Voss (2009).

Adicional, según Freije et al. (2021), la CS desempeña un papel relevante en la prestación de servicio debido a su carácter relacional lo cual ayuda al desarrollo de ventajas competitivas, donde las empresas altamente servitizadas tienen un mayor nivel de innovación.

En la alineación: las estrategias, procesos y capacidades de una unidad funcional son consistentes con una o más unidades funcionales adicionales dentro de la organización; para actuar de manera 
coherente y utilizar plenamente sus recursos disponibles (Sombultawee y Boon-itt, 2018, p.1), estando sujetas a dos tipos básicos de restricciones: la demanda de sus bienes y servicios (el marketing); costo, capacidad y pericia de sus recursos (la gestión de operaciones). Por lo que, la coordinación estrecha entre ambas es un elemento importante y necesario del éxito empresarial (Dixon et al., 2014).

Las estrategias de marketing $y$ operaciones integradas son cruciales para generar un resultado positivo en la experiencia de compra de los consumidores, por ejemplo: en la configuración física del ambiente donde se presta un servicio de la empresa (Bitner, 1992; Chase y Jacobs, 2019).

La integración marketing-operaciones ha sido un tema que ha llamado la atención por su relevancia en la competitividad de la compañía; sin embargo; en la práctica aún se requiere entender cómo se integran y aplicarlas adecuadamente. Aún en muchas compañías estas relaciones son hostiles y difíciles, impactando negativamente en la satisfacción del cliente y la competitividad (Sombultawee y Boon-itt, 2018).

Según Sombultawee y Boon-itt (2020), un sistema de evaluación y recompensa, o de alineación de incentivos $y$ recompensas apropiado favorece la promoción de la colaboración entre departamentos.

Altuntas (2015), propuso combinar la perspectiva de marketing con GCSS. Tal marco beneficiaría en términos de alinear sus actividades de red de suministro con la demanda existente de productos y servicios sostenibles. Sin embargo, en este campo existen oportunidades para futuras investigaciones para generalizar y determinar su impacto en la competitividad, adicional a que los mayores esfuerzos se han realizado en la industria manufacturera.

En la literatura se han observado estudios sobre la alineación de las interfaces marketing-operaciones $y$ marketing-GCS, de forma separada. Sin embargo, Pero y Lamberti (2013) destacan que la interfaz marketing-GCS tiene un trasfondo de operaciones; por lo que se puede concluir que aun existiendo adecuada alineación marketingoperaciones, el servicio de entrega y abastecimiento puede fallar en satisfacer los requerimientos o expectativas del cliente cobrando importancia la interfaz marketing-GCS. Entonces, con enfoque principal en el área de marketing, y de acuerdo con Golgeci y Gligor (2017) sobre las posibles sinergias entre marketing y otras funciones, se puede deducir que es posible que la interfaz marketing-AOCS produzca mayores beneficios que sus interfaces aisladas, al tener una visión más integrada y así poder reducir mayor cantidad de conflictos y ser de mayor impacto positivo en la competitividad de la empresa, en su desempeño operativo y estratégico (Attia, 2021), con el uso eficiente de dicha interfaz marketing-AOCS.

Se requiere la alineación de objetivos y actividades organizacionales, conciencia interfuncional, y adoptar un enfoque 
holístico para la creación y entrega de valor (Golgeci y Gligor, 2017), de esta forma se puede promover la cooperación en la interfaz marketing-AOCS de manera integral. Más que usar un sistema de recompensas o incentivos para la alineación de esta interfaz, se debe considerar todos los aspectos incluidos en los trabajos de: Ávila et al., (2019); Bardhan y Pattnaik (2017); Bijmolt, et al. (2021); Goh y Eldridge (2019); Golgeci y Gligor (2017); Kwortnik y Thompson (2009); y Piercy (2010). Porque las medidas de cooperación, si son indirectas y basadas en resultados, suelen ser inadecuadamente implementadas como lo observó Sombultawee y Boon-itt (2020).

De esta manera, se propone un sistema más completo y engranado que se pueda ajustar al contexto organizacional $\mathrm{y}$ que genere el mayor impacto positivo posible en la competitividad de la empresa usando de manera eficiente la interfaz marketing-AOCS, en lugar de alineaciones multifuncionales aisladas (marketing-operaciones; marketingGCS).

Se puede asumir, por los estudios revisados (Altuntas, 2015: Bijmolt, et al., 2021; De Souza et al., 2016; Freije et al., 2021; Gölgeci y Kuivalainen, 2020), que involucran la CS (verde, sostenible y resiliente) y los estudios de marketingoperaciones (Dixon et al., 2014; Samuel et al., 2017; Sombultawee y Boon-itt, 2018; y Vicenzi et al., 2020); adicional a los estudios de Golgeci y Gligor (2017) y de Pero y Lamberti (2013); que al trabajar de manera integral la interfaz
marketing-AOCS, la experiencia del cliente en el servicio será de mayor impacto en las ventas, así como en la recompra, utilizando en este caso la lógica inductiva.

Dado que no existen estudios anteriores que evidencien esta interfaz marketing-AOCS para servicios, existe la oportunidad de futuras investigaciones para evaluar y medir su impacto, así como revisar sistemas que promuevan su adecuada alineación.

\section{Conclusiones}

El papel de marketing-AOCS debe ser altamente enfatizado dentro de la organización y abordado deliberadamente por el más alto nivel de liderazgo, más aún en servicios donde la relación es más importante considerando que se diseña y ejecuta una oferta de servicios centrada prácticamente en la experiencia del cliente; siguiendo lo enunciado por Dixon et al. (2014).

Los microambientes de marketing han sido afectados por una serie de cambios y muchas de las operaciones empresariales se han visto modificadas por el distanciamiento social provocado por la pandemia, muchas de estas alteraciones probablemente generen una nueva forma de trabajo y de comportamiento del consumidor (He y Harris, 2020; Kwok, et al., 2020; y Wang et al., 2020), lo que podría producir nuevas oportunidades de encuentro para la interfaz marketingAOCS.

Goh y Eldridge (2019) encontraron 
que un procedimiento PVO altamente formalizado o estandarizado inhibe el desempeño de la CS. Dixon et al. (2014) indicaron que no se comprende aún si el nivel de colaboración (marketingoperaciones) genera adicionales distracciones y presión sobre los gerentes. Golgeci y Gligor (2017) indican que se requiere la alineación de objetivos y actividades organizacionales, así como adoptar un enfoque holístico para la creación y entrega de valor; por lo que se puede deducir que la estrategia de trabajo y alineación usada en la interfaz marketing-AOCS impacta la competitividad positiva o negativamente, y que lo indicado por Golgeci y Gligor (2017) sobre las posibles sinergias entre marketing y otras funciones cobra mayor probabilidad.

Se observó que los mecanismos usados para propiciar la alineación de las interfaces, por lo general recaen en la organización y en la arquitectura efectiva de interacciones con el mercado (Noci, 2019).

Existe un campo abierto en la investigación en cuanto a entender en la práctica cómo se integran adecuadamente marketing-AOCS para servicios, la promoción de la alineación interfuncional y su impacto.

Es importante fomentar estratégicamente la cooperación entre marketing-AOCS por medio de un sistema holístico que involucre tanto a marketing, administración de operaciones y la GCS; ajustándolo al contexto organizacional, y siendo lo suficientemente flexible para permitir la toma decisiones oportuna; así también, promoviendo el involucramiento del personal de los diferentes niveles jerárquicos, en el ámbito de sus competencias; con la intención de generar el mayor impacto positivo posible en la competitividad de la empresa y la satisfacción de los clientes internos y externos.

La investigación aporta con los resultados de los análisis realizados sobre las actuales interfaces multifuncionales entre marketing y los componentes que forman la AOCS (operaciones y GCS) y que se proponen trabajar en forma integrada, en lugar de alineaciones multifuncionales aisladas (marketingoperaciones; marketing-GCS); dependiendo del enfoque de marketing y el contexto organizacional; logrando un engranaje eficiente, por cuanto es posible deducir que al trabajar de manera integrada marketing-AOCS la experiencia del cliente en el servicio será de mayor impacto en las ventas, así como en la recompra, y los conflictos y restricciones en las interacciones funcionales se pueden disminuir y gestionar de forma más eficaz.

\section{Contribución de autores}

A.N.R. Revisión de literatura, metodología, análisis de datos, discusión y conclusiones conjuntas, revisión de redacción.

J.C.C. Revisión de literatura, metodología, análisis de datos, discusión y conclusiones conjuntas, revisión de redacción. 


\section{Referencias}

Ailawadi, K., y Farris, P. (2017). Managing multi and omni-channel distribution: Metrics and research directions. Journal of Retailing, 93(1), 120-135. http:// dx.doi.org/10.1016/j.jretai.2016. 12.003

Akdoğan, M., y Durak, A. (2016). Logistic and Marketing Performances of Logistics Companies: A Comparison between Germany and Turkey. Procedia - Social and Behavioral Sciences, 235, 576-586. https://doi.org/10.1016/j.sbspro.2016.11. 084

Altuntas, C. (2015). Sustainable Demand Chain Management: An Alternative Perspective for Sustainability in the Supply Chain, Procedia - Social and Behavioral Sciences, 207, 262-273. https:// doi.org/10.1016/j.sbspro.2015.10.095

Attia, A. (2015). Testing the effect of marketing strategy alignment and triple-A supply chain on performance in Egypt. EuroMed Journal of Business, 10(2), 163-180. https://doi.org/10.1108/EMJB-07-2014-0 020

Ávila, P., Lima, D., Moreira, D., Pires, A., y Bastos, J. (2019). Design of a Sales and Operations Planning (S\&OP) process case study. Procedia CIRP, 81, 1382-1387. https://doi.org/10.1016/j.pro cir.2019.04.048

Bardhan, A., y Pattnaik, S. (2017). Effect of crossfunctional integration between operations and marketing on negative critical incidents. Total Quality Management \& Business Excellence, 28(11-12), 1357-1377. https://doi.org/10.1080/1478 3363.2016.1147943

Bhoompag, M., Sambrani, S., y Prabhukumar, A. (2017). Emerging trends in green marketing: challenges, opportunities and strategies in indian perspective. International Journal of Science
Technology and Management, 6(3), 485-490. Recuperado de http://www. ijstm.com/images/short_pdf/1490683400 _S3158.pdf

Bijmolt, T., Broekhuis, M., de Leeuw, S., Hirche, C., Rooderkerk, R., Sousa, R., y Zhu, S. (2021). Challenges at the marketing-operations interface in omni-channel retail environments. Journal of Business Research, 122, 864-874. https://doi.org/10.1016/j.jbus res.2019.11.034

Bitner, M. (1992). Servicescapes: The Impact of Physical Surroundings on Customers and Employees. Journal of Marketing, 56(2), 57-71. https://doi.org/10.2307/1252042

Chase, R., y Jacobs F. (2019). Administración de Operaciones Producción y Cadena de Suministros (15a ed.). México: Mc Graw Hill Education.

Chowdhury, M., Sarkar, A., Saha, P., y Anik, R. (2020). Enhancing supply resilience in the COVID-19 pandemic: a case study on beauty and personal care retailers. Modern Supply Chain and Applications, 2(3), 143-159. https://doi.org/10. 1108/MSCRA-07-2020-0018

De Souza, E., Giro, R., y Caldeira, A. (2016). Green Marketing as a Mediator Between Supply Chain Management and Organizational Performance. RAM. Revista de Administração Mackenzie, 17(3), 183-211. https://dx.doi.org/10.1590/167 8-69712016/administracao.v17n3p183-211

Dixon, M., Karniouchina, E., van der Rhee, B., Verma, R., y Victorino, L. (2014). The role of coordinated marketing-operations strategy in services: Implications for managerial decisions and execution. Journal of Service Management, 25(2), 275-294. https://doi.org/10.1108/JOSM02-2014-0060

El Ouardighi, F., Feichtinger, G., y Fruchter, G. 
(2018). Accelerating the diffusion of innovations under mixed word of mouth through marketing-operations interaction. Annals of Operations Research, 264, 435-458. https:// doi.org/10.1007/s10479$017-2649-2$

Finger, M., Lacerda, D., Camargo, L., Piran, F., Cassel, R., y Morandi, M. (2021). Impacts of marketing decisions on delivery performance and flexibility of the operations area, International Journal of Productivity and Performance Management, Vol. ahead-of-print No. ahead-of-print. https://doi.org/10.1108/ IJPPM-05-2020-0238

Freije, I., de la Calle, A., y Ugarte, J. (2021). Role of supply chain integration in the product innovation capability of servitized manufacturing companies. Techno vation.102216. https://doi.org/10.1016/j. technovation. 2020.102216

Fu, C., Cheng, S., y Yi, Y. (2019). Dynamic Control of Product Innovation, Advertising Effort, and Strategic Transfer-Pricing in a Marketing-Operations Interface. Mathematical Problems in Engineering, 2019, 1-14. https://doi.org/10.1155/2019/8418260

Goh, S., y Eldridge, S. (2019). Sales \& Operations Planning: The Effect of Coordination Mechanisms on Supply Chain Performance. International Journal of Production Economics, 214, 80-94. https://doi.org/10.1016/j.ijpe.2019.03.027

Golgeci, I., y Gligor, D. (2017). The interplay between key marketing and supply chain management capabilities: the role of integrative mechanisms. Journal of Business \& Industrial Marketing, 32(3), 472-483. https://doi.org/10.1108/JBIM05-2016-0102

Gölgeci, I., y Kuivalainen, O. (2020). Does social capital matter for supply chain resilience? The role of absorptive capacity and marketing-supply chain management alignment. Industrial Marketing Management, 84, 63-74. https://doi.org/ 10.1016/j.indmarman.2019.05.006

He, H., y Harris, Ll. (2020). The impact of Covid-19 pandemic on corporate social responsibility and marketing philosophy. Journal of Business Research, 116 , 176-182. https://doi.org/10.1016/j.jbus res.2020.05.030

Hernández-Sampieri, R., Cuevas, A., Mendoza, C., y Méndez, S. (2017). Fundamentos de investigación. McGraw-Hill.

Kim, R. (2020). The Impact of COVID-19 on Consumers: Preparing for Digital Sales. IEEE Engineering Management Review, 48(3), 212-218. https://doi.org/10.1109/ EMR.2020.2990115

Kwok, L., Tang, Y., y Yu, B. (2020). The 7 Ps marketing mix of home-sharing services: Mining travelers' online reviews on Airbnb. International Journal of Hospitality Management, 90. https: //doi.org/10.1016/j.ijhm.2020.102616

Kwortnik, R., y Thompson, G. (2009). Unifying services marketing and operations with service experience management. Journal of Service Research, 11(4), 389-406. https://doi.org/10.1177/1094670509333595

Le, K., y Massey, G. (2019). Improving relationships between sales and marketing: the relative effectiveness of cross-functional coordination mechanisms. Journal of Marketing Management, 35(13-14), 1267-1290. https://doi.org/10.1080/02 67257X.2019.1648310

Leppelt, T., Foerstl, K., y Hartmann, E. (2013). Corporate Social Responsibility in Buyer-Supplier Relationships: Is it Beneficial for Top- Tier Suppliers to Market their Capability to Ensure a Responsible Supply Chain? Business Research, 6, 126- 152. https://doi.org/10. 1007/BF03342746 
Lozano, R., y Barreiro-Gen, M. (2021). Disrupting the brave new world: COVID-19 effects on organizations' sustainability efforts. Journal of Organizational Change Management, 34(3), 613- 628. https://doi.org/10.1108/JOCM-09-2020-0 276

Marques, A., Lacerda, D., Riehs, L., y Teixeira, R. (2014). Exploring the relationship between marketing and operations: Neural network analysis of marketing decision impacts on delivery performance. International Journal of Production Economics, 153, 178-190. https://doi.org/10.1016/j.ijpe.2014.02.020

Maynard, M., Falcone, E., Petersen, K., Fugate, B., y Bonney, L. (2020). Conflicting paradigms in manufacturing and marketing decisions: The effects of situational awareness on team performance. International Journal of Production Economics, 230. https:// doi.org/10.1016/j.ijpe.2020.107801

Noci, G. (2019). The evolving nature of the marketing-supply chain management interface in contemporary markets. Business Process Management Journal, 25(2), 379-383. https://doi.org/10.1108/ BPMJ-07-2018-0202

O’Connor, N., Lowry, P., y Treiblmaier, H. (2020). Interorganizational cooperation and supplier performance in high- technology supply chains. Heliyon 6(3), e03434 https://doi.org/10.1016/j.heliyon.2020.e0 3434

Patel, J., y Malpani, A. (2020). Impact of Covid19 on e-marketing: An Early Stage Review International Journal of Advanced Science and Technology, 29(12s), 1774-1779. Recuperado de http://sersc. org/journals/index.php/IJAST/article/vie w/23918/12474

Pero, M., y Lamberti, L. (2013). The supply chain management-marketing interface in product development: An exploratory study. Business Process Management Journal, 19(2), 217-244. https://doi.org/ $10.1108 / 14637151311308295$

Piercy, N. (2010). Improving marketing-operations cross-functional relationships. Journal of Strategic Marketing, 18(4), 337-356. https://doi.org/10.1080/09652541003768 095

Prestes, M., Teixeira, J., Patricio, L., y Sangiorgi, D. (2019). Leveraging service design as a multidisciplinary approach to service innovation. Journal of service Management 30(6), 681- 715. https:// doi.org/10.1108/JOSM-07-2017-0178

Raddats, C., Kowalkowski, C., Benedettini, O., Burton, J., y Gebauer, H. (2019). Servitization: A contemporary thematic review of four major research streams. Industrial Marketing Management, 83, 207-223. https://doi.org/10.1016/j.indmar man.2019.03.015

Samuel, R., Mesak, H., e Inman, R. (2017). A dynamic marketing-operations interface model of new product updates. European Journal of Operational Research, 257(1), 233-242. https://doi.org/10.1016/j.ejor.20 16.07.051

Shahid, S., y Khan, S. (2016). Integrating Operations and Marketing in the Fast Food Delivery Industry. The Lahore Journal of Business, 4(2), 23-50. Recuperado de https:// lahoreschoolofeconomics.edu.pk/busines sjournals/V4issue2/02\%20Shahid\%20an d\%20Khan\%20FINAL.pdf

Sombultawe, K. (2017). Marketing - operations alignment: Scale Development and validation (dissertation for the degree of Doctor of Philosophy Business Administration). Thammasat University. Ref. code: 25605602320045AOM. Recuperado de http://ethesisarchive. library.tu.ac.th/thesis/2017/TU_2017_56 02320045_9135_8673.pdf 
Sombultawee, K., y Boon-itt, S. (2018). Marketingoperations alignment: A review of the literature and theoretical background. Operations Research Perspectives, 5, 1-12. https://doi.org/10.1016/j.orp.2017. 11.001

Sombultawee, K., y Boon-itt, S. (2019). Marketing operations alignment: A systematic literature and citation network analysis review. The Kasetsart Journal of Social Sciences, 40(1), 225-234. https://doi.org/ 10.1016/j.kjss.2017.09.009

Sombultawee, K., y Boon-itt, S. (2020). Marke ting-Operations Alignment: Scale Development and Validation. Journal of Business \& Industrial Marketing, 35(12), pp. 2079-2095. https://doi.org/10.1108/ JBIM-09-2019-0431

Veeralakshmi, M. (2020). Green marketing: innovative mantra of marketing. International Journal of Advanced Science and Technology, 29(7s), 1967-1981. Recuperado de http://sersc. org/journals/index.php/IJAST/article/vie $\mathrm{w} / 12284$

Vicenzi, J., Antoni, V., Damacena, C., Riss, C., y Manosso, T. (2020). Effects of coexistence of marketing and operations on product innovation performance. Gestão \& Produção, 27(1), e4039. https://doi.org/10.1590/0104-530x4039-20

Vorre, A. (2019). Value co- creation in service marketing: A critical (re)view. International Journal of Innovation Studies, 3(4), 73-83. https://doi.org/ $10.1016 /$ j.ijis.2019.12.001

Wang, Y., Hong, A., Li, X., y Gao, J. (2020). Marketing innovations during a global crisis: A study of China firms' response to COVID-19. Journal of Business Research, 116, 214- 220. https://doi.org/ 10.1016/j.jbusres.2020.05.029

Yang, J., Xie, H., Yu, G., y Liu, M. (2020).
Antecedents and consequences of supply chain risk management capabilities: an investigation in the post-coronavirus crisis. International Journal of Production Research, 59(5), 1573-1585. https://doi.org/10.1080/00207543.2020.1 856958

Yu, W., Jacobs, M., Chávez, R., y Feng, M. (2017). The impacts of IT capability and marketing capability on supply chain integration: a resource based perspective. International Journal of Production Research, 55(14), 4196-4211. DOI:10. $1080 / 00207543.2016 .1275874$

Yu, W., Ramanathan, R., y Nath, P. (2014). The impacts of marketing and operations capabilities on financial performance in the UK retail sector: A resource-based perspective. Industrial Marketing Management, 43(1), 25-31. https://doi. org/10.1016/j.indmarman.2013.07.014

Zanon, C., Filho, A., Jabbour, C., y Jabbour, A. (2013). Alignment of operations strategy: exploring the marketing interface. Industrial Management and Data Systems, 113(2), 207-233. https:// doi.org/10.1108/02635571311303541

Zomerdijk, L., y Voss, C. (2009). Service design for experience-centric services, Journal of Service Research, 13(1), 67-82. https:// doi.org/10.1177/1094670509351960 